\title{
Radiofrequency thermocoagulation in cases of atypical trigeminal neuralgia: a retrospective study
}

\author{
Mert Akbas ${ }^{1}$, Haitham Hamdy Salem ${ }^{2}$ (D), Tamer Hussien Emara² ${ }^{2}$ Bora Dinc ${ }^{1}$ and Bilge Karsli ${ }^{1}$
}

\begin{abstract}
Background: Trigeminal neuralgia (TN) is the most common cause of neuropathic facial pain. Atypical TN are usually refractory to conventional medical treatments and the standard microvascular decompression surgeries that lead to marked negative impact on the patient's quality of life.

Objective: The aim of this study is to examine the efficacy and safety of radiofrequency (RF) ablation in cases with atypical TN that did not respond to standard medical treatments.

Methods: Our study included 19 consecutive patients with refractory atypical TN for more than 6 months. Neurological examination and MRI brain did not reveal any significant correlated signs. RF thermocoagulation was performed under fluoroscopy guidance. Collected data included preoperative conditions, pre- and post-procedure visual analog scale (VAS) score, and complications. Patients were followed up for a period of 1-year duration to evaluate the long-term efficiency of the procedure.

Results: A total of 19 patients were recruited into the study ( $56.32 \pm 13.48$ years, $21 \%$ males, $79 \%$ females). Females outnumbered males (15 female patients, 79\%) versus (4 male patients, 21\%). There was more than 50\% improvement in VAS score (16 patients, $79 \%$ versus 3 patients, $21 \%)(p<0.05)$. Age was the only statistically significant factor were more elderly patients showed better improvement regarding VAS score $(p<0.05)$.

Conclusions: Radiofrequency thermocoagulation is an effective and relatively safe procedure for alleviating atypical facial pain.

Keywords: Efficacy, Radiofrequency thermocoagulation, Safety, Atypical trigeminal neuralgia, Complication, Visual analog scale
\end{abstract}

\section{Introduction}

Trigeminal neuralgia (TN) is the most common cause of neuropathic facial pain, with an annual incidence of 3-5 new cases per 100,000 population [1]. Idiopathic TN is classified to typical and atypical forms based on the clinical manifestations. Atypical cases usually have continuous pain between the classic paroxysmal lancinating attacks [2]. Atypical TN cases are usually less responsive to conventional medical treatments like carbamazepine or oxcarbazepine and the standard microvascular decompression surgeries [3]. This increases the social, psychological, and occupational burden of the disease.
There are different treatment modalities in cases with refractory TN including microvascular decompression, Gamma Knife surgeries, and radiofrequency (RF) procedures [4-6]. RF is less invasive and safe, with immediate results and minimal side effects apart from facial numbness, which is usually accepted by the patients [7].

Patients with typical TN usually respond to RF ablation [7-13]. Yet, limited studies have addressed the efficacy of RF in cases with atypical trigeminal neuralgia $[14,15]$. The aim of this study is to examine the effects of RF ablation in cases with atypical TN that did not respond to standard medical treatments.

\footnotetext{
* Correspondence: haithamhamdy@yahoo.com

${ }^{2}$ Department of Neurology, Ain Shams Univeristy, Cairo, Egypt

Full list of author information is available at the end of the article
} 


\section{Patients and methods}

We retrospectively studied patients who received RF thermocoagulation for treating medication resistant atypical TN from January 2014 through December 2017 in the pain management department, Akdeniz University, Antalya. The study was approved by the Akdeniz University Local Research Ethics Committee. We collected the following data from our electronic medical record: patient demographics, neurological examination, MRI findings, pain scores before and after the procedure (Visual analogue scale), and improvement after the procedure (scored at 0 to $100 \%$ ). The primary outcome measure was a $50 \%$ reduction in the severity of pain measured by VAS score. Patients were followed up for a period of 1-year duration to evaluate long-term efficacy of the procedure. Patients who did not respond were offered a second procedure to relieve their symptoms.

Our study included 19 patients with atypical TN [2]. Inclusion criteria were patients above 18 years old, not responding to carbamazepine or oxcarbazepine for not less than 6 months duration or were intolerant to medications side effects. Neurological examination did not reveal any significant correlated signs apart of some sensory deficit along mandibular and/or maxillary nerve distribution. Brain MRI of all patients did not show any clinically relevant abnormalities. Exclusion criteria included typical TN, presence of any neurological signs or MRI findings suggestive of secondary cause, and history of previous surgical interventions for trigeminal neuralgia.

\section{Procedure}

Prior to the RF thermocoagulation procedure, each patient underwent a prognostic nerve block using $0.25 \%$ bupivacaine HCL (Marcaine-Astra Zeneca). Those who showed $>50 \%$ improvement in pain were eligible for RF thermocoagulation. During the RF procedure, the head of every patient was fixed, and no sedation was administered in order to be able to distinguish the affected branch of the trigeminal nerve. All the patients were cannulated, and vital data monitoring was performed throughout the procedure. The injection site was sterilized and draped.

The foreman ovale was identified with a C-arm fluoroscopy. The skin at the entry point was infiltrated with lidocaine HCL (Jetokain-Adeka) and a 2-mm active-ended RF electrode (Trigeminal RF electrode $2 \mathrm{~mm}$-Cosman) was inserted and advanced parallel to the axis of the fluoroscopy till passing the foramen ovale border (Fig. 1). Aspiration was done. Test stimulation was applied at $2 \mathrm{~Hz}, 0.1-$ $1.5 \mathrm{~V}$ for motor stimulation and $50-100 \mathrm{~Hz}, 0.1-1.5 \mathrm{~V}$ for sensory stimulation with observing movement and sensory changes described by the patient in the corresponding dermatome. After confirming the position of the electrode, radiofrequency thermocoagulation was applied at $70^{\circ} \mathrm{C}$ for $90 \mathrm{~s}$, then the needle was removed, and an ice pack was applied on the face. The patient was kept under observation for $2 \mathrm{~h}$.

\section{Data analysis}

Analysis was done in SPSS Version 22 (IBM Corporation, Armonk, NY) using one-way ANOVA for continuous outcome variables, the Pearson correlation

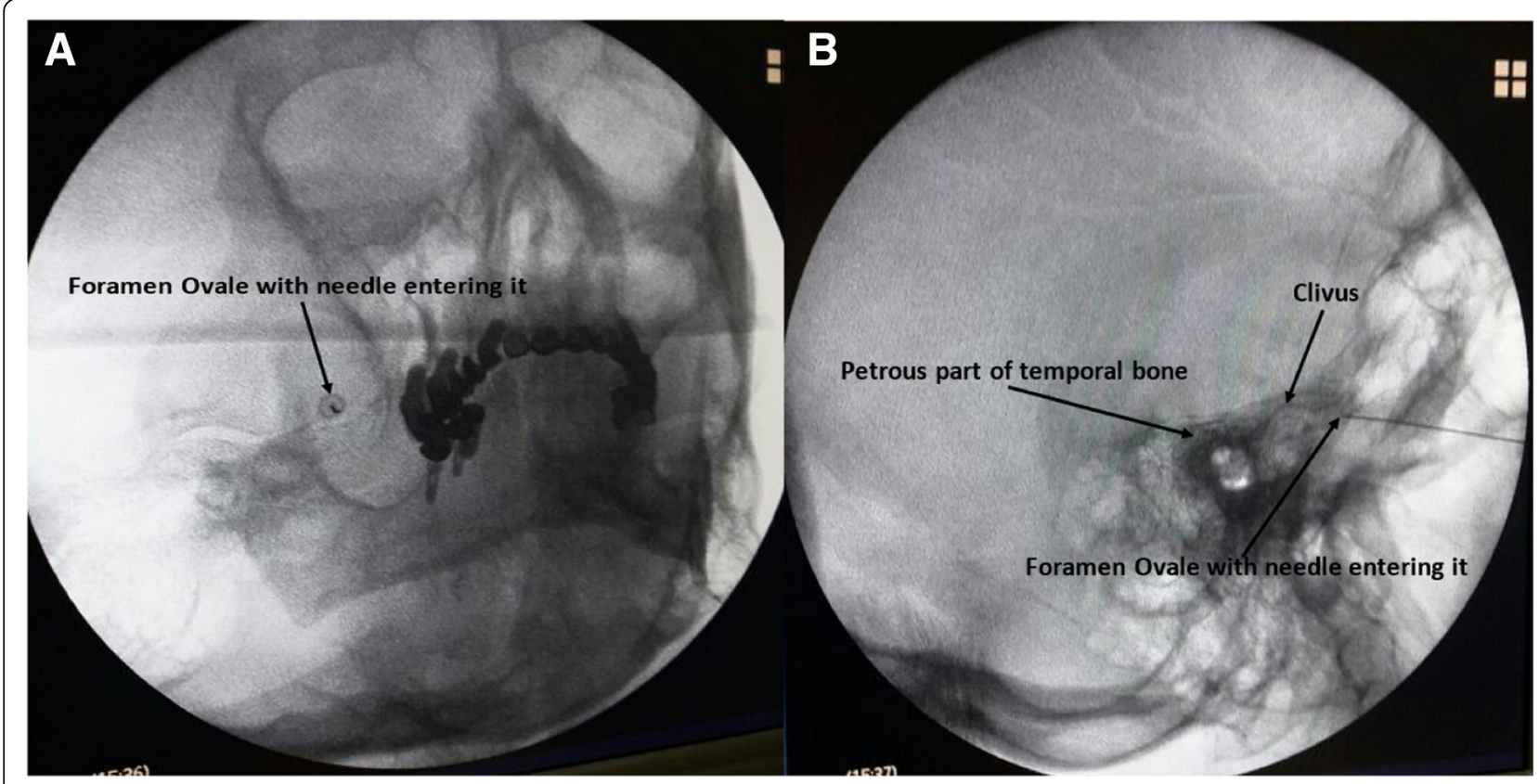

Fig. 1 a Submental view of the foramen ovale and $\mathbf{b}$ lateral view to confirm the depth of the needle insertion 
coefficient, and the $\chi^{2}$ test for dichotomous outcome variables.

\section{Results}

A total of 19 patients were recruited into the study. Females outnumbered males ( 15 female patients, $79 \%$ ) versus (4 male patients, 21\%). Most of our studied population (16 patients, $79 \%$ ) had right-sided trigeminal pain. Other patient characteristics are shown in Table 1.

There was more than $50 \%$ improvement in VAS score (16 patients, 79\% versus 3 patients, $21 \%)(p<0.05)$. Age was the only statistically significant factor. We found that older patients showed a better improvement regarding VAS score $(p<0.05)$. On the contrary, sex, the side of the trigeminal pain, and the trigeminal nerve branch and/or number affected revealed no statistical significance $(p>0.05)$.

Regarding complications, only one patient (5\%) developed mild facial edema that subsided spontaneously within a few days, yet none of the studied patients developed any major adverse events.

\section{Discussion}

Radiofrequency thermocoagulation has been proven to be safe and effective in treating different chronic pain disorders. It has been widely used in the treatment of refractory TN in the past decade as early pain relief can be attained in $98 \%$ of patients [4].

In our study, atypical TN was statistically more common among females (16 patients, 79\%). Gender was proven to play an important role in the incidence of TN as female predominance was demonstrated by many reviewers $[1,16,17]$. Our patients' peak age of disease onset was between the sixth and seventh decades of life.

Table 1 General characteristics of the study population

\begin{tabular}{ll}
\hline Gender & $N=19$ \\
Men & $4(21 \%)$ \\
Women & $15(79 \%)$ \\
Age & $56.32 \pm 13.48$ \\
Side of pain & \\
Right & $13(68.5 \%)$ \\
Left & $6(31.5 \%)$ \\
Branches affected & \\
Mandibular + maxillary & $6(31.5 \%)$ \\
Mandibular & $7(37 \%)$ \\
Maxillary & $6(31.5 \%)$ \\
Number of procedures & \\
One & $16(84 \%)$ \\
Two & $3(16 \%)$ \\
\hline
\end{tabular}

This trend was confirmed by multiple previous studies. Accordingly, it is fundamental to perform thorough neurological assessment to exclude associated diseases in patients under 40 years of age [1, 18-21]. Using MRI, a study documented radiologically that women and elderly patients have significantly smaller trigeminal nerve volumes compared to men and young candidates which can be considered as a cofactor for TN [22].

In our study population, the right side was more affected than the left (13 patients, $68.5 \%$ versus 6 patients, $31.5 \%$, respectively). This may be attributed to the anatomical fact that foramen rotundum and foramen ovale are usually narrower on the right side [16, 23-25]. The mandibular and/or maxillary branches were equally affected. This disease predilection pattern conforms with that of other studies [16, 17, 26, 27].

$\mathrm{RF}$ is the most common percutaneous procedure used to treat typical TN and offers the highest rate of efficiency (90-100\%) and complete pain relief [4, 28-37]. Similarly, in our study, RF showed high efficiency $(p<$ $0.05)$ in pain relief in 16 patients and only 3 patients (21\%) did not reach our required $>50 \%$ pain improvement in VAS score. Few previous case reports revealed similar results in cases of atypical TN $[14,15]$.

Statistical analysis revealed a significant relationship between post RF pain relief and age, as older patients showed higher improvement in pain severity following the procedure. To our knowledge, there are no studies that evaluate this relationship but most studies recommend RF as the first line of treatment for medication refractory TN $[5,8,14,27]$.

Our study was limited by the number of patients and would benefit from a larger sample. Including more pain and patient's satisfaction scores would be favorable in confirming the results.

In conclusion, this study suggests that RF thermocoagulation is an effective and relatively safe procedure for alleviating atypical facial pain. If confirmed in other studies, this will have important implications for the management of patients with atypical TN.

Acknowledgements
N/A
Funding
None

Availability of data and materials

Available on request.

\section{Authors' contributions}

MA participated in the design of the study, performed some of the procedures for radiofrequency thermocoagulation, and revised the manuscript. HHS performed some of the procedures for radiofrequency thermocoagulation and participated in the manuscript production. THE participated in the data analysis and participated in the manuscript production. BD took part in the data collection and participated in the data 
analysis. BK participated in the design of the study and revised the manuscript. All authors read and approved the final manuscript.

\section{Ethics approval and consent to participate}

Based upon regulations of Akdeniz university ethical committee, ethics approval and consents were not needed as this study was retrospective and all data were de-identified.

\section{Consent for publication}

N/A

\section{Competing interests}

The authors declare that they have no competing interests.

\section{Publisher's Note}

Springer Nature remains neutral with regard to jurisdictional claims in published maps and institutional affiliations.

\section{Author details}

'Deparment of Pain Medicine, Akdeniz Univeristy, Antalya, Turkey.

${ }^{2}$ Department of Neurology, Ain Shams Univeristy, Cairo, Egypt.

Received: 5 January 2019 Accepted: 10 June 2019

Published online: 01 July 2019

\section{References}

1. Katusic S, et al. Incidence and clinical features of trigeminal neuralgia, Rochester, Minnesota, 1945-1984. Ann Neurol. 1990;27(1):89-95.

2. Cruccu G, et al. Trigeminal neuralgia: new classification and diagnostic grading for practice and research. Neurology. 2016;87(2):220-8.

3. Bick SKB, Eskandar EN. Surgical treatment of trigeminal neuralgia. Neurosurg Clin N Am. 2017;28(3):429-38.

4. Emril DR, Ho KY. Treatment of trigeminal neuralgia: role of radiofrequency ablation. J Pain Res. 2010;3:249-54.

5. Eugene AR. Trigeminal neuralgia and radiofrequency lesioning. Brain (Bacau). 2015;6(1-2):91-6.

6. Al-Quliti KW. Update on neuropathic pain treatment for trigeminal neuralgia. The pharmacological and surgical options. Neurosciences (Riyadh). 2015;20(2):107-14.

7. Huang Q, et al. The effectiveness and safety of thermocoagulation radiofrequency treatment of the ophthalmic division (V1) and/or maxillary (V2) and mandibular (V3) division in idiopathic trigeminal neuralgia: an observational study. Pain Physician. 2016;19(7):E1041-7

8. Yao $P$, et al. Efficacy and safety of continuous radiofrequency thermocoagulation plus pulsed radiofrequency for treatment of V1 trigeminal neuralgia: a prospective cohort study. Medicine (Baltimore). 2016; 95(44):e5247.

9. Motamedi $\mathrm{MH}$, et al. Trigeminal neuralgia and radiofrequency. J Calif Dent Assoc. 2009;37(2):109-14

10. Motamedi $\mathrm{MH}$, et al. Trigeminal neuralgia and radiofrequency. Todays FDA 2010;22(5):54-5, 57-9

11. Tang $Y Z$, et al. Long-term outcome of computed tomography-guided percutaneous radiofrequency thermocoagulation for classic trigeminal neuralgia patients older than 70 years. J Craniofac Surg. 2014;25(4):1292-5.

12. Tang $Y Z$, et al. The long-term effective rate of different branches of idiopathic trigeminal neuralgia after single radiofrequency thermocoagulation: a cohort study. Medicine (Baltimore). 2015:94(45):e1994.

13. Zhao YX, et al. Trigeminal somatosensory-evoked potential: a neurophysiological tool to monitor the extent of lesion of ganglion radiofrequency thermocoagulation in idiopathic trigeminal neuralgia: a case-control study. Medicine (Baltimore). 2017:96(3):e5872.

14. Nguyen $M$, Wilkes D. Pulsed radiofrequency V2 treatment and intranasal sphenopalatine ganglion block: a combination therapy for atypical trigeminal neuralgia. Pain Pract. 2010;10(4):370-4.

15. Du S, et al. Ophthalmic branch radiofrequency thermocoagulation for atypical trigeminal neuralgia:a case report. Springerplus. 2015:4:813.

16. Cruccu G, et al. Diagnosis of trigeminal neuralgia: a new appraisal based on clinical and neurophysiological findings. Suppl Clin Neurophysiol. 2006:58: 171-86

17. Hall GC, et al. Epidemiology and treatment of neuropathic pain: the UK primary care perspective. Pain. 2006;122(1-2):156-62.
18. Harris W. Three cases of alcohol injection of the Gasserian ganglion for trigeminal neuralgia. Proc R Soc Med. 1912;5(Clin Sect):115-9.

19. Harris W. Bilateral destruction of Gasserian ganglia by alcohol injection for chronic trigeminal neuralgia. Proc R Soc Med. 1920;13(Clin Sect):62-4.

20. Ullik R. Results of alcohol injection into the gasserian ganglion in trigeminal neuralgia. Wien Med Wochenschr. 1958;108(45-46):933-7.

21. Kalyanaraman S, Ramamurthi B. Trigeminal neuralgia--a review of 331 cases. Neurol India. 1970;18(Suppl 1):100-8.

22. Kress $B$, Schindler $M$, Rasche $D$. Why do advanced age and female gender predispose to trigeminal neuralgia? Clin Neurol. 2008;18(1):60-4.

23. Neto HS, Camilli JA, Marques MJ. Trigeminal neuralgia is caused by maxillary and mandibular nerve entrapment: greater incidence of rightsided facial symptoms is due to the foramen rotundum and foramen ovale being narrower on the right side of the cranium. Med Hypotheses. 2005:65(6):1179-82.

24. Larsen A, et al. Trigeminal neuralgia: diagnosis and medical and surgical management. JAAPA. 2011:24(7):20-5.

25. Reinard K, et al. Racial disparities in the diagnosis and management of trigeminal neuralgia. J Neurosurg. 2017;126(2):368-74.

26. MacDonald BK, et al. The incidence and lifetime prevalence of neurological disorders in a prospective community-based study in the UK. Brain. 2000; 123(Pt 4):665-76.

27. Hunt K, Patwardhan R. Trigeminal neuralgia: a modern-day review. Int Rev Neurobiol. 2007;79:621-31.

28. Erdine $\mathrm{S}$, et al. Comparison of pulsed radiofrequency with conventional radiofrequency in the treatment of idiopathic trigeminal neuralgia. Eur J Pain. 2007;11(3):309-13.

29. Zakrzewska JM, Akram H. Neurosurgical interventions for the treatment of classical trigeminal neuralgia. Cochrane Database Syst Rev. 2011;9:CD007312.

30. Li X, et al. A prospective study of Gasserian ganglion pulsed radiofrequency combined with continuous radiofrequency for the treatment of trigeminal neuralgia. J Clin Neurosci. 2012;19(6):824-8.

31. Akbas $M$, et al. Sphenopalatine ganglion pulsed radiofrequency treatment in patients suffering from chronic face and head pain. Braz J Anesthesiol. 2016 66(1):50-4.

32. Abd-Elsayed $\mathrm{A}$, et al. Pulsed radiofrequency for treating trigeminal neuralgia. Ochsner J. 2018:18(1):63-5.

33. Ali Eissa AA, et al. The efficacy and safety of combined pulsed and conventional radiofrequency treatment of refractory cases of idiopathic trigeminal neuralgia: a retrospective study. J Anesth. 2015;29(5):728-33.

34. Elawamy A, Abdalla EEM, Shehata GA. Effects of pulsed versus conventional versus combined radiofrequency for the treatment of trigeminal neuralgia: a prospective study. Pain Physician. 2017;20(6):E873-81.

35. Huang $Y$, et al. Percutaneous radiofrequency thermocoagulation for the treatment of different types of trigeminal neuralgia: evaluation of quality of life and outcomes. J Huazhong Univ Sci Technol Med Sci. 2010;30(3):403-7.

36. Liu YG, et al. Selective radiofrequency thermocoagulation for trigeminal neuralgia. Zhonghua Yi Xue Za Zhi. 2010;90(29):2059-61.

37. Yao $P$, et al. Radiofrequency thermocoagulation for V2N3 idiopathic trigeminal neuralgia: effect of treatment temperatures on long-term clinical outcomes: a cohort study. Medicine (Baltimore). 2016:95(26):e4019.

\section{Submit your manuscript to a SpringerOpen ${ }^{\circ}$ journal and benefit from:}

- Convenient online submission

- Rigorous peer review

- Open access: articles freely available online

- High visibility within the field

Retaining the copyright to your article

Submit your next manuscript at $>$ springeropen.com 